\title{
Feasibility of Placement of an Anterior Cervical Epidural Blood Patch for Spontaneous Intracranial Hypotension
}

J.K. Park and J.P. Villablanca

\begin{abstract}
SUMMARY: The dorsal epidural blood patch is a commonly used management technique for spontaneous intracranial hypotension from a dural CSF leak, but it may be less efficacious for cervical or ventral leaks. We report the technique of placing an anterior cervical blood patch for a large cervical ventral leak. To our best knowledge, this approach has not been reported. In the appropriately selected patient, an anterior cervical epidural blood patch may be safely used.
\end{abstract}

ABBREVIATIONS: EBP = epidural blood patch; $\mathrm{IH}=$ intracranial hypotension; $\mathrm{SIH}=$ spontaneous $\mathrm{IH}$

H

is a clinical condition typically characterized by orthostatic headaches secondary to CSF hypovolemia. ${ }^{1}$ While headaches exacerbated by standing and relieved by lying down are classically described, nausea and neck stiffness are also frequently associated with $\mathrm{IH}$, among other symptoms. ${ }^{2}$ Although the most common cause of secondary IH is lumbar puncture, IH secondary to spine surgery, overdrainage by neurosurgical procedures, and degenerative spine disease are also described. ${ }^{1}$ Primary or SIH is also well described. ${ }^{1}$ Despite the term "spontaneous," patients frequently recall a history of trivial trauma, suggesting a mechanical role ${ }^{1,2}$; connective tissue disorders have also been described as a risk factor. ${ }^{3,4}$

The diagnosis of SIH can be established by several methods, including brain MR imaging, myelography with MR imaging or $\mathrm{CT}$, lumbar puncture, and radionuclide cisternography. ${ }^{2}$ Dural tears causing SIH may occur anywhere throughout the spine, and while some cases may resolve with conservative therapy, several treatment options exist for patients with persistent symptoms. A common treatment for SIH is the EBP, in which autologous blood is injected into the spinal epidural space at the site of the suspected dural defect. ${ }^{1,2}$ Directed cervical EBP injections have been described by multiple authors for dural leaks localized to the cervical spine, ${ }^{5-7}$ as has a bilateral transforaminal cervicothoracic approach. ${ }^{8}$ While the standard technique of EBP is a dorsal approach, an extensive data base search revealed that no prior re-

Received April 12, 2012; accepted after revision June 2.

From the Department of Neuroradiology, Ronald Reagan UCLA Medical Center, Los Angeles, California.

Please address correspondence to J. Pablo Villablanca, MD, Department of Radiology, UCLA Medical Center, 757 Westwood Plaza, Los Angeles, CA, 90095; e-mail: pvillablanca@mednet.ucla.edu.

http://dx.doi.org/10.3174/ajnr.A3244 ports describing an anterior approach to the EBP for the treatment of SIH exist to date. We present the technique of placing an EBP via an anterior transcervical approach in a patient with refractory headache secondary to SIH from a ventral cervical dural leak.

\section{TECHNIQUE AND ILLUSTRATIVE CASE}

A 41-year-old man presented 1 year after the onset of severe positional headaches that began when he was suddenly picked up from behind by the chest and agitated by a friend. Four months before headache onset, he also described a hang gliding accident during which he struck the ground with his head at approximately 50 miles per hour; he was neurologically intact and denied headaches at that time. His headaches subsequently progressed to the point of occasional loss of consciousness, and he was unable to work. While his headaches initially were alleviated by lying supine and analgesics and coffee, relief was eventually only achieved by placing his head lower than body level in a sitting position. Walking and sitting up exacerbated symptoms significantly.

Before presentation at our medical center, he was diagnosed with multifocal ventral cervical and thoracic CSF leaks by CT myelography. He was subsequently treated unsuccessfully with 2 separate lumbar epidural blood patches at outside hospitals, in addition to chiropractic treatments. He then presented to our institution for further evaluation and underwent an additional CT myelography, which revealed a large and continuous ventral epidural contrast collection extending from the $\mathrm{C} 3-\mathrm{C} 4$ through T11 levels (Figs 1 and 2). While the exact site of communication with the subarachnoid space could not be identified, both a contrast-attenuation gradient and the largest cross-sectional area of the epidural collection were seen in the lower cervical region, suggesting that the site of leakage was in the cervical spine. Radio- 


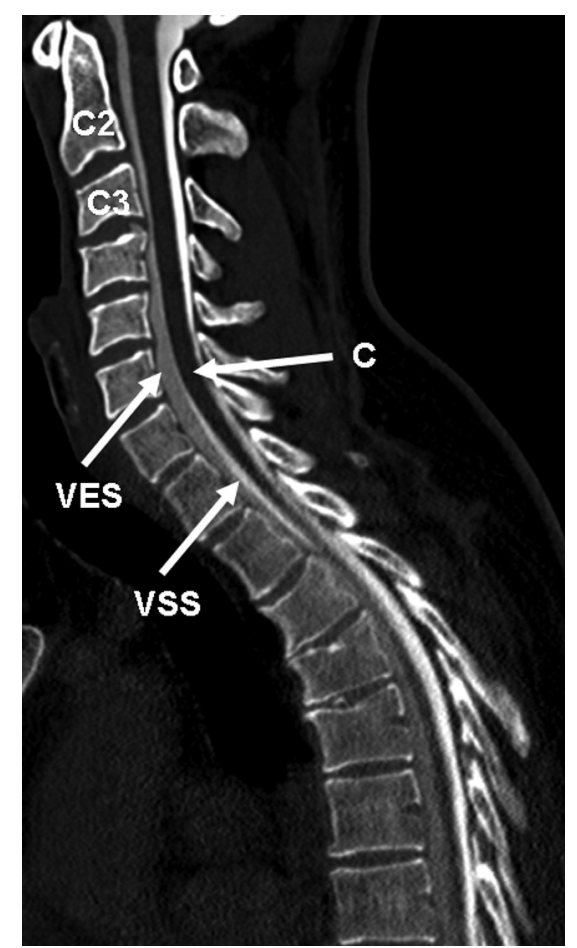

FIG 1. Sagittal postmyelogram CT of the cervical and upper thoracic spine showing a ventral epidural contrast collection. VES indicates ventral epidural space; VSS, ventral subarachnoid space; C, cord.

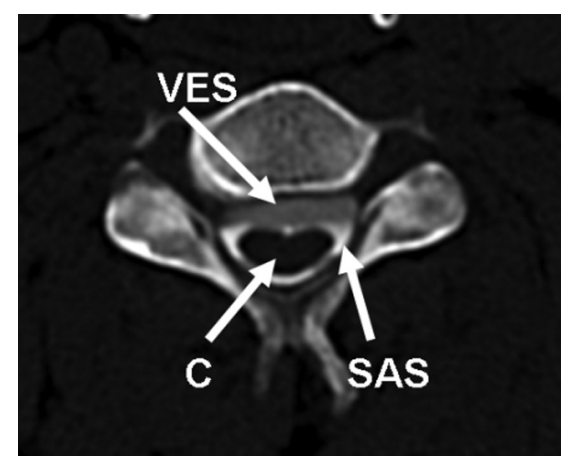

FIG 2. Axial postmyelogram CT through the cervical spine demonstrating ventral epidural contrast collection. SAS indicates subarachnoid space.

nuclide CSF study by using indium-111 diethylene triamine pentaacetic acid was subsequently ordered to further localize the site of leakage but revealed no CSF leak. Neurosurgery was consulted, but given the large size of the fluid collection and unknown exact location of dural injury, it was thought that exploring the ventral spinal column would require a morbid surgery and extensive vertebrectomies. A consultation for possible EBP subsequently occurred.

Given the estimated size and ventral location of the CSF collection, it was decided to attempt an anterior EBP approach. The patient was placed supine on the fluoroscopy table. Following sterile anterior neck preparation and conscious sedation with vital sign monitoring, the C5-C6 disk space was identified by using frontal fluoroscopy. Local anesthesia was provided with $1 \%$ lidocaine. The patient was instructed to relax the muscles of the neck and shoulders, as gradually increasing digital pressure over the

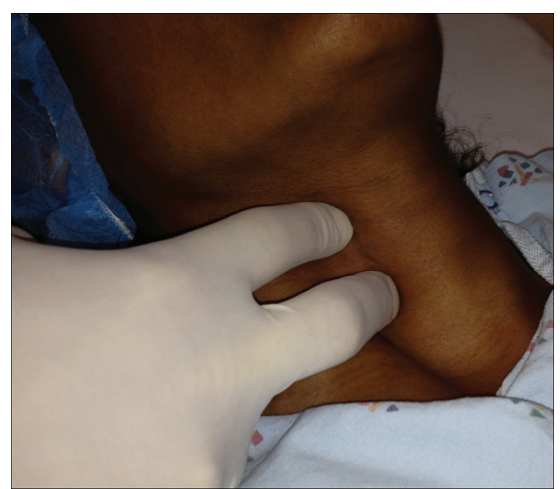

FIG 3. Demonstration of the technique of digital palpation of the neck and manual displacement of critical structures such as the common carotid artery. The right side is generally preferred because the esophagus usually descends left of midline.

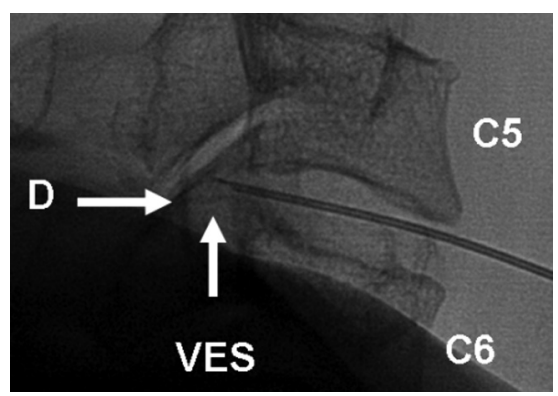

FIG 4. Lateral fluoroscopic image demonstrating the needle in the C5-C6 disk space by using a right anterior slightly oblique approach. D indicates dura.

midcervical region was applied in the right anterior neck by using the index and middle fingers (Fig 3). The fingers were used to displace the right carotid sheath laterally, and the esophagus and trachea medially. A left-sided approach was avoided because the cervical esophagus generally descends left of midline. Downward digital pressure was continued until the fingertips contacted the firm and slightly undulating contour of the prevertebral fascia, without the presence of palpable yielding or pulsatile intervening soft-tissue structures.

Next, while maintaining constant digital contact with the prevertebral fascia and maintaining the interdigital access window by using the opposite hand, we advanced a 3.5-inch 22-ga spinal needle into and through the midportion of the target disk by using anteroposterior fluoroscopy, with entry just to the right of midline, aiming at the posterior center of the disk. These precautions were taken to protect and avoid vital structures ventral to the cervical spine, specifically the common carotid artery, vertebral artery, internal jugular vein, vagus nerve, pharynx, and trachea. Lateral fluoroscopy was used to ascertain the depth of the slow needle advance, with the tip terminating in the ventral epidural space $2 \mathrm{~mm}$ beyond the posterior vertebral body margin (Fig 4).

We used 0.2-mL of iohexol (Omnipaque 240; GE Healthcare, Princeton, New Jersey) to confirm needle tip position within the ventral collection (Fig 1). Gentle aspiration yielded $10 \mathrm{~mL}$ of clear CSF without resistance. A second injection of $0.2 \mathrm{~mL}$ of Omnipaque 240 again outlined the ventral epidural collection, which had decreased in thickness from approximately $6.6 \mathrm{~mm}$ anteroposteriorly preaspiration to $3.3 \mathrm{~mm}$ postaspiration. A total of 6 
$\mathrm{mL}$ of fresh sterile peripheral venous blood was then obtained from an antecubital fossa vein. Following an approximate wait time of 4 minutes, the blood was slowly injected into the ventral epidural cervical collection. The 4-minute period was used on the basis of visual analysis; at this time, the blood visually demonstrated early clotting but was still able to be expelled from the syringe. Subsequently a lumbar drainage catheter was placed at the L 4 level, and the patient underwent 72 hours of lumbar drainage at $5 \mathrm{~mL}$ per hour while recumbent. The patient reported immediate relief of his headaches following the procedure and remained neurologically intact throughout the procedure and drainage period.

At 3-month follow-up, a repeat myelogram revealed persistent leakage of myelographic dye into the cervicothoracic ventral epidural space without significant change in collection dimensions. Similar findings were noted at the 14-month follow-up as well. However, the patient described significant clinical improvement throughout the 14-month period. His main complaint was interscapular pain, and he stated he was mostly headache-free, except when placing his head below the level of his knees while sitting in a chair.

\section{DISCUSSION}

Our patient developed intractable and progressive headaches secondary to a high-volume ventral dural tear in the lower cervical region; he may even have had a multifocal or fast-flowing leak. Although the exact site of the leak could not be established despite repeat imaging, it was presumed to be large due to the extensive size of the collection. We postulate that he initially failed treatment with lumbar EBPs at the outside hospitals due to the cervical and ventral location of his dural CSF leak and the presumed large size of his dural tear.

While the dorsal interlaminar loss-of-resistance technique in placing an EBP is the standard approach, a dorsal cervical blood patch was not considered for this ventral leak due to concern that the volume of blood necessary to seal the ventral leak would also result in spinal cord compression. Placing an EBP far from the level of a leak was a possibility, but reported results are mixed. ${ }^{9,10}$ Given that this patient was not an ideal operative candidate due to the nonlocalized nature of his leak and previous failed treatment with lumbar EBP, it was thought that a directed approach was reasonable.

The amount of blood injected can be proportional to the amount of CSF aspirated from the epidural collection but should be limited in total volume if the dural leak is suspected to be large or in wide-open communication with the subarachnoid space, to avoid entry of a large amount of blood into the epidural space. Doing so could help prevent possible iatrogenic complications such as spinal cord compression, vasospasm, and superficial siderosis. Close neurologic monitoring was provided in this case during and for 72 hours after the procedure. A lumbar drainage catheter was also placed to minimize the hydrostatic pressure gradient across the site of leakage.
Despite the patient's abnormal myelographic findings following anterior cervical EBP placement, he reported only mild residual symptoms throughout the 14 months following his procedure. Although he demonstrated significant symptomatic improvement following anterior EBP placement, because of his persistent abnormal imaging findings, the positive clinical outcome is not definitively attributable to the EBP. The possibility that postprocedural lumbar drainage contributed to the patient's clinical improvement is not excluded either. Although relapsing and remitting courses are seen in patients with dural leaks, given the substantial follow-up period of 14 months with consistently reported improvements in severe symptoms, we do not believe the patient's improvements were simply attributable to a relapsing and remitting course. Despite the discordance between imaging changes and clinical improvement, in this report, we emphasize the technical feasibility and safety of placing an anterior cervical EBP.

\section{CONCLUSIONS}

We present, to our knowledge, the first technical report of the placement of an anterior EBP for the treatment of SIH from a cervical ventral dural tear. We suggest that the anterior technique can be safely performed in patients with ventral cervical leaks. While we very slowly injected a total of $6 \mathrm{~mL}$ of fresh peripheral blood, the ideal volume for anterior injections warrants further investigation.

\section{REFERENCES}

1. Inamasu J, Guiot BH. Intracranial hypotension with spinal pathology. Spine J 2006;6:591-99

2. Schievink WI. Spontaneous spinal cerebrospinal fluid leaks. Cephalalgia 2008;28:1345-56

3. Schievink WI, Gordon OK, Tourje J. Connective tissue disorders with spontaneous spinal cerebrospinal fluid leaks and intracranial hypotension: a prospective study. Neurosurgery $2004 ; 54: 65-70$, discussion 70-71

4. Mokri B, Maher CO, Sencakova D. Spontaneous CSF leaks: underlying disorder of connective tissue. Neurology 2002;58:814-16

5. Kantor D, Silberstein SD. Cervical epidural blood patch for low CSF pressure headaches. Neurology 2005;65:1138

6. Allegri M, Lombardi F, Custodi VM, et al. Spontaneous cervical (C1C2) cerebrospinal fluid leakage repaired with computed tomography-guided cervical epidural blood patch. J Pain Symptom Manage 2010;40:e9-e12

7. Usui T, Saito S, Goto F. Spontaneous intracranial hypotension treated with a cervical epidural blood patch. Eur J Anaesthesiol 2003;20:500-02

8. Walega D, McComb E, Rosenow J. Bilateral cervicothoracic transforaminal blood patches for persistent headache from spontaneous intracranial hypotension: a case report and review. Clin J Pain 2011;27:357-64

9. Su CS, Lan MY, Chang YY, et al. Clinical features, neuroimaging and treatment of spontaneous intracranial hypotension and magnetic resonance imaging evidence of blind epidural blood patch. Eur Neurol 2009;61:301-07

10. Sencakova D, Mokri B, McClelland RL. The efficacy of epidural blood patch in spontaneous CSF leaks. Neurology 2001;57:1921-23 